\title{
A new Lorentz violating model with particle's "maximum energy"
}

Jinwen Hu ( $\sim$ 2007.hujinwen@163.com )

Department of Physics and Technology, Wuhan University, Wuhan 430060, China

\section{Research Article}

Keywords: Lorentz model, ultrahigh energy, Gamma ray burst, rainbow model, variable speed of light, time lag

Posted Date: January 13th, 2021

DOl: https://doi.org/10.21203/rs.3.rs-144454/v1

License: (9) This work is licensed under a Creative Commons Attribution 4.0 International License. Read Full License 


\title{
A new Lorentz violating model with particle's "maximum
}

\author{
energy" \\ Jinwen $\mathrm{Hu}^{1 *}$ \\ ${ }^{1}$ Department of physics and technology, Wuhan university, Wuhan 430060, China
}

\begin{abstract}
In this paper we re-investigated the relationship between the symmetry of inertial systems and the Lorentz transformation. We found that when we just follow the following three principles: (1)we can define the time in the whole space with a prescribed clock synchronization, (2)the time-space is uniform and the space is isotropic and (3)all the inertial systems are equivalent, then we can totally construct a general coordinate transformation to meet the symmetry of inertial systems, and with a special assumption on the speed of light, we can construct a non-Lorentz transformation between inertial systems to make the particle's energy have a limited value, which is similar to the rainbow model.

Similar to the usual Lorentz violating models, the non-Lorentz transformation in this paper lead to a new modified disperse relation. We applied the obtained disperse relation to analyze the photon's arrival time lag effect in astronomy and found that the "maximum energy" derived in our model is somewhat related to the "maximum energy" assumed in the rainbow model.
\end{abstract}

\section{Keywords}

Lorentz model; ultrahigh energy; Gamma ray burst; rainbow model; variable speed of light; time lag

\section{Introduction}

It is well known that the Special Relativity and General Relativity, implying the Lorentz invariance, have already made great achievements, but at the same time the Lorentz violating models are also of some astrophysical interest. In the past few decades the scientific community has shown an intense interest in the theories that contained and investigated the breakdown of Lorentz symmetry in many scenarios [1-5] and also the so-called Deformed Special Relativities (DSR) [6]. For example, in this scenario of considering the combination of Quantum theory and Gravity theory, the rainbow model is proposed, which introduces the "minimum length" as another constant between inertial systems besides the speed of light. In the framework of rainbow model, the particle's energy is finite rather than be infinite, and correspondingly, the particle's energy-momentum dispersion relation is modified as

\footnotetext{
* Corresponding author, E-mail: 2007.hujinwen@163.com
} 
the following form (set $c=1)[7,8]$

$$
\left[1+\chi_{1}\left(\frac{E}{E_{L V}}\right)^{1}+\chi_{2}\left(\frac{E}{E_{L V}}\right)^{2}+O\left(\frac{E}{E_{L V}}\right)^{3}\right] E^{2}-\boldsymbol{p}^{2}=m_{0}^{2}
$$

where $E$ denotes the total energy of the particle, $m_{0}$ denotes the rest mass, $\boldsymbol{p}$ is the momentum, and $E_{\mathrm{LV}}$ denotes the energy scale at which Lorentz violating effects become strong, the couplings $\chi_{s}= \pm 1(s=1,2)$ are determined by the dynamical framework being studied.

The rainbow model provides a new perspective for the development of Quantum Gravity. For example, the application of Eq. (1) can make the black hole's temperature to be convergence $[9,10]$ and alleviate the singularity problem arose in the Big Bang model $[11,12]$, etc. The rainbow model have made progress in solving some problems with extreme conditions, but the specific form of rainbow model is still unknown. Therefor, a lot of work has been done to determine the parameters involved in the rainbow model. In the previous work, for example, Bolmont [13] applied the HETE-2 gamma ray burst data to constrain the energy scale $E_{\mathrm{LV}}$ to be at least $2 \times 10^{15}$ $\mathrm{GeV}$ at 95\% CL, Nilsson [14] applied the Hubble+SNIa+BAO (BOSS+Lyman a) $+\mathrm{CMB}$ data to constrain $E_{\mathrm{LV}}$ to be at least the order of $10^{16} \mathrm{GeV}$ at $1 \sigma$, or even $10^{17}$ $\mathrm{GeV}$ at $3 \sigma$. And based on the first-order approximation of Eq. (1), $\mathrm{Xu}[15,16]$ analyzed the GRB 160509A event and claimed that there exists a linear relation between the variable speed of light and the photon's energy, and $E_{\mathrm{LV}} \approx 3.6 \times 10^{17} \mathrm{GeV}$ was obtained.

However, the rainbow model is somewhat complicated, especially the correction terms of Eq. (1) are still unclear in physics. But the rainbow model is a special and typical Lorentz violating model, it inspired us that there may be other possible relationship between the speed of light and the symmetry of inertial systems. So this paper re-investigated the relationship between the symmetry of inertial systems and the Lorentz model. We found that by imposing some new special rules on the speed of light, we can construct a general function on the speed of light to make the inertial systems to be equivalent, which is shown in the content of Sect. 2. In Sect. 3, by taking a specific assumption on the speed of light, we obtained a non-Lorentz transformation between inertial systems to make the particle's energy have a limited value, which is similar to the rainbow model. In Sect. 4, we applied the modified energy-momentum dispersion relation derived from the non-Lorentz transformation to analyze the photon's arrival time lag effect in astronomy, and found that the particle's "maximum energy" derived in our paper is somewhat related to the "maximum energy" assumed in the rainbow model. In Sect. 5 we summarized the paper.

\section{Variable speed of light}

It is well known that the rainbow model presents that the speed of light maybe associated with the photon's energy $[15,16]$, then here we proposed a general hypothesis that: For a light source in vacuum, when it moves at a velocity $v$ relative to an observer in vacuum, then the observed (by the observer) speed of light emitted by the light source is $n c$, where $n$ is a dimensionless quantity, $c$ is the speed of light in vacuum. Obviously, in order not to violate some fundamental principles and experiments, we should impose some rules on the parameter $n$ as follows

1. Firstly, as stated in the special relativity, we should define the (proper) time (note that the only time that makes sense in special relativity is the proper time 
measured by the clocks carried by physical observers) in the whole space with a prescribed clock synchronization, that is, for a specific inertial system, based on the speed of light emitted by a light source that fixed in the specific inertial system, we can calibrate the clock fixed in the inertial system to synchronize. So it requires that

$$
n(v=0, c)=1
$$

2. Secondly, according to the general concept of time and space that the time-space is uniform and the space is isotropic, it requires that $n(v, c)$ is independent of the direction of vector $v$ and $c$, namely,

$$
n(v, c)=n(-v, c)=n(v,-c)=n(-v,-c)
$$

3. In addition, we also follow the principle that all the inertial systems are equivalent.

Based on the above assumptions on the speed of light, next we will derive the coordinate transformation between the two inertial systems $S(\boldsymbol{x}, \boldsymbol{y}, \boldsymbol{z}, t)$ and $S^{\prime}\left(\boldsymbol{x}^{\prime}, \boldsymbol{y}^{\prime}, \boldsymbol{z}^{\prime}, t^{\prime}\right)$. And here we assume that $S^{\prime}$ is moving at a velocity $v$ relative to $S$.

Firstly, for simplicity, we assume that the three spatial coordinates of the two coordinate systems are parallel to each other, and the direction of $\boldsymbol{v}$ is along the $\boldsymbol{x}$-axis or $\boldsymbol{x}^{\prime}$-axis, then we obtain $\boldsymbol{y}^{=} \boldsymbol{y}^{\prime}, \boldsymbol{z}=\boldsymbol{z}^{\prime}$.

Secondly, since the time-space is uniform, the coordinate transformation between $S$ and $S^{\prime}$ should be in a linear form (note that in the rainbow model, the coordinate transformation between $S$ and $S^{\prime}$ is assumed to be in a nonlinear form, here we follow that the time-space is uniform and thus the coordinate transformation between $S$ and $S^{\prime}$ should be in a linear form), and we assume that

$$
\boldsymbol{x}=\gamma\left(\boldsymbol{x}^{\prime}+\boldsymbol{v} t^{\prime}\right)
$$

Where $\gamma=\gamma(\boldsymbol{v}, \boldsymbol{c})$ is a proportionality factor.

The inverse transformation of Eq. (4) is

$$
\boldsymbol{x}^{\prime}=\gamma^{\prime}[\boldsymbol{x}+(-\boldsymbol{v}) t]
$$

Where $\gamma^{\prime}=\gamma(-v)$ is a proportionality factor.

Based on the symmetry of space, we will obtain

$$
\gamma(\boldsymbol{v}, \boldsymbol{c})=\gamma(-\boldsymbol{v},-\boldsymbol{c})
$$

Note that the above we didn't distinguish the direction of vector $v$ and $c$, i.e., the direction of vector $\boldsymbol{v}$ and $\boldsymbol{c}$ maybe along the positive $\boldsymbol{x}$-axis (or $\boldsymbol{x}^{\prime}$-axis) or along the negative $\boldsymbol{x}$-axis (or $\boldsymbol{x}^{\prime}$-axis). And if we distinguish the direction of vector $\boldsymbol{v}$ and $\boldsymbol{c}$ by the positive and negative signs, we will obtain four different combinations, i.e., $(v, c)$, $(v,-c),(-v, c),(-v,-c)$. Based on Eq. (6) we can obtain $\gamma(v, c)=\gamma(-v,-c), \gamma(-v, c)=\gamma(v$, $-c)$.

Now we will solve the expression for $\gamma$. If the light signal is emitted by the light source at the moment that the origin of $S$ and $S^{\prime}$ are coincides, then based on the above assumption on the speed of light, we will obtain 


$$
\left\{\begin{array} { l } 
{ x ^ { 2 } + y ^ { 2 } + z ^ { 2 } = ( c t ) ^ { 2 } } \\
{ x ^ { \prime 2 } + y ^ { \prime 2 } + z ^ { \prime 2 } = ( n c t ^ { \prime } ) ^ { 2 } } \\
{ y = y ^ { \prime } = 0 } \\
{ z = z ^ { \prime } = 0 }
\end{array} \quad \text { and } \quad \left\{\begin{array}{l}
x^{\prime 2}+y^{\prime 2}+z^{\prime 2}=\left(c t^{\prime}\right)^{2} \\
x^{2}+y^{2}+z^{2}=(n c t)^{2} \\
y=y^{\prime}=0 \\
z=z^{\prime}=0
\end{array}\right.\right.
$$

The first formula of Eq. (7) represents that when the light source is fixed in $S$, then for the observer in $S$, the observed speed of light is $c$ (based on Eq. (2)), while for another observer in $S^{\prime}$, the observed speed of light is $n c$.

Similarly, since $S$ and $S^{\prime}$ are symmetric, when the light source is fixed in $S$, then for the observer in $S$, the observed speed of light is $c$, while for another observer in $S$, the observed speed of light is $n c$, which case corresponds to the second formula of Eq. (7).

Based on Eqs. (4) (7), and similar to the derivation method shown in the special relativity theory, we can solve the expression for $\gamma$ and obtain the coordinate transformation between $S$ and $S^{\prime}$

$$
\left\{\begin{array}{l}
\boldsymbol{x}^{\prime}=\gamma(\boldsymbol{x}-\boldsymbol{v} t) \\
t^{\prime}=\gamma\left(t-\frac{\boldsymbol{v}}{k^{2}(\boldsymbol{v}, \boldsymbol{c})} \boldsymbol{x}\right)
\end{array}\right.
$$

where $\gamma(v, c)=1 / \sqrt{1-v^{2} / k^{2}}, k(v, c)=\sqrt{n v c^{2} /(n c-c+v)}$.

From Eq. (8) it can be seen that $k(v, c)=k(-v,-c), k(-v, c)=k(v,-c)$, and $\gamma(v, c)=\gamma(-v,-c), \gamma(-v, c)=\gamma(v,-c)$, which is agree with Eq. (6).

Here it should be noted that, from Eqs. (4) (7), it doesn't require that $k(v, c)$ is equal to $k(-v, c)$. In fact $k(v, c)$ and $k(-v, c)$ represent the two different cases or two independent events, they cannot be linked together by the symmetry in one event.

Based on Eq. (8) we can obtain

$$
\frac{d \boldsymbol{x}^{\prime}}{d t^{\prime}}=\frac{d \boldsymbol{x}-\boldsymbol{v} d t}{d t-\frac{\boldsymbol{v}}{k^{2}} d \boldsymbol{x}}=\frac{d \boldsymbol{x} / d t-\boldsymbol{v}}{1-\frac{\boldsymbol{v}}{k^{2}(\boldsymbol{v}, \boldsymbol{c})} \frac{d \boldsymbol{x}}{d t}}=f(\boldsymbol{v}, \boldsymbol{c})
$$

As stated above that the direction of vector $v$ and $c$ maybe along the positive $\boldsymbol{x}$-axis (or $\boldsymbol{x}^{\prime}$-axis) or along the negative $\boldsymbol{x}$-axis (or $\boldsymbol{x}^{\prime}$-axis), and if we distinguish the direction of vector $\boldsymbol{v}$ and $\boldsymbol{c}$ by the positive and negative signs, we will obtain four different combinations, i.e., $(v, c),(v,-c),(-v, c),(-v,-c)$. Then based on Eq. (9), we can obtain that

Case 1: Note that the above assumed that $S^{\prime}$ is moving at a velocity $v$ relative to $S$. When the light source is fixed in $S$, then for the observer in $S$, the observed speed of light is $c$, while for the observer in $S^{\prime}$, it has 


$$
\left\{\begin{array}{l}
\text { when } \frac{d x}{d t}=c, \frac{d x^{\prime}}{d t^{\prime}}=f(v, c)=\frac{c-v}{1-\frac{v}{k^{2}(v, c)} c}=f(-v, c)=\frac{c+v}{1-\frac{-v}{k^{2}(-v, c)} c}=n c \\
\text { when } \frac{d x}{d t}=-c, \frac{d x^{\prime}}{d t^{\prime}}=f(v,-c)=\frac{-c-v}{1-\frac{v}{k^{2}(v,-c)}(-c)}=f(-v,-c)=\frac{-c+v}{1-\frac{-v}{k^{2}(-v,-c)}(-c)}=-n c
\end{array}\right.
$$

Case 2: Similarly, since $S$ and $S^{\prime}$ are equivalent, when the light source is fixed in $S^{\prime}$, then for the observer in $S^{\prime}$, the observed speed of light is $c$, while for the observer in $S$, it has (note that in this case the velocity of $S$ relative to the light source is $-v$ )

$$
\left\{\begin{array}{l}
\frac{d x}{d t}=\frac{d \boldsymbol{x}^{\prime} / d t^{\prime}-(-\boldsymbol{v})}{1-\frac{-\boldsymbol{v}}{k^{2}(-\boldsymbol{v}, \boldsymbol{c})} \frac{d \boldsymbol{x}^{\prime}}{d t^{\prime}}}=f^{\prime}(\boldsymbol{v}, \boldsymbol{c}) \\
\text { when } \frac{d x^{\prime}}{d t^{\prime}}=c, \frac{d x}{d t}=f^{\prime}(v, c)=\frac{c+v}{1+\frac{v}{k^{2}(-v, c)} c}=f^{\prime}(-v, c)=\frac{c-v}{1+\frac{-v}{k^{2}(v, c)} c}=n c \\
\text { when } \frac{d x}{d t}=-c, \frac{d x}{d t}=f^{\prime}(v,-c)=\frac{-c+v}{1+\frac{v}{k^{2}(-v,-c)}(-c)}=f^{\prime}(-v,-c)=\frac{-c-v}{1+\frac{-v}{k^{2}(v,-c)}(-c)}=-n c
\end{array}\right.
$$

Eq. (10) and Eq. (11) can be also expressed in a vector form

$$
\left\{\begin{array}{l}
\text { when } \frac{d \boldsymbol{x}}{d t}=c, \frac{d \boldsymbol{x}^{\prime}}{d t^{\prime}}=n c \\
\text { when } \frac{d \boldsymbol{x}^{\prime}}{d t^{\prime}}=\boldsymbol{c}, \frac{d \boldsymbol{x}}{d t}=n c
\end{array}\right.
$$

Eq. (12) shows all the meaning of the speed of light, and it also shows that Eq. (8) is just the solution of Eq. (7).

Importantly, it can be seen that the forms of Eq. (8) are similar to the Lorentz transformation, i.e., replacing $c$ in the Lorentz transformation with $k$ we can obtain Eq. (8), and similar to the proof method shown in the special relativity, it is easy to prove that the Maxwell's Equations are also covariant based on Eq. (8).

Based on Eq. (8) we can obtain the time-space metric

$$
d s^{2}=-k^{2} d t^{2}+d x^{2}
$$

Correspondingly, the particle's energy-momentum dispersion relation is

$$
E^{2}=\boldsymbol{p}^{2} k^{2}+E_{0}^{2}
$$

Where $E_{0}=m_{0} k^{2}$ denotes the particle's energy, $E=\gamma m_{0} k^{2}$ is the total energy of the particle, $\boldsymbol{p}=\gamma m_{0} \boldsymbol{v}$ denotes the particle's momentum.

Here it should be noted that, similar to the other Lorentz violating models, at above we just try to allow the speed of light to be possible to vary between inertial 
systems, and subsequently we try to impose some constraints on the parameter $n$ to make the time-space meet the above three principles, thus we obtain a self-consistent coordinate transformation between inertial systems. The idea is different from the DSR model, which introduce a second constant besides the speed of light, and also different from the Standard Model Extension (SME) model, which assumed there exists extra fields resulting in the observer Lorentz transformation is reserved while the particle Lorentz transformation is violated. In this paper we didn't introduce any constants or fields, and the speed of light observed by the different observers is not constant, but we obtained a special non-Lorentz model. And the forms of the coordinates transformation, the particle's energy-momentum dispersion relation and the time-space metric obtained in this paper are similar to which obtained from the Lorentz model.

\section{Particle's "maximum energy"}

As we know, in Lorentz model, the particle's energy tends to be infinite when the particle's velocity is close to the speed of light, however, the DSR model introduces a new constant as the energy limit of the particles, which is considered to be necessary in the theory of Quantum Gravity. In this paper, as Eq. (8) shown that if $n \equiv 1$ then Eq. (8) returns to the Lorentz model. But here we would like to discuss another interesting case where $n$ is not always equal to 1 , as the general Lorentz violating models suggested [1-6].

Inspired by the idea of rainbow model, we found that Eq. (8) imply that it is possible the particle's energy have a limit.

Based on Eq. (8), the time-space scaling factor is

$$
\gamma=\frac{1}{\sqrt{1-v^{2} / k^{2}}}=\frac{1}{\sqrt{\frac{1-v / c}{n}\left(n+\frac{v}{c}\right)}}
$$

Eq. (15) inspires us that when $\boldsymbol{v}=\boldsymbol{c}$, it is possible that $\gamma$ does not tend to be infinite if we assume $n=0$ when $\boldsymbol{v}=\boldsymbol{c}$. So here we can construct an expression for $n$. Since $n$ has been constrained in Eq. (2) and Eq. (3), that is

$$
\left\{\begin{array}{l}
n(v=0, c)=1 \\
n(v, c)=n(v,-c)=n(-v, c)=n(-v,-c) \\
\lim _{v \rightarrow c} \frac{1-v / c}{n}=\text { const }
\end{array}\right.
$$

Based on Eq. (16), we can only take a few but finite kinds of expressions for $n$, and the following expression for $n$ is one of them

$$
n=\frac{1}{1-Q}\left(1-Q^{1-v^{2} / c^{2}}\right)
$$

where $Q$ is a constant.

Besides Eq. (17) one may consider another expression for $n$, but as we know 
there are many experiments restricting the violation of Lorentz model, so we should choose an expression for $n$ to not violate the previous experiments' results. Figure 1 shows the $n \sim v$ curve when taking $Q=(\mathbf{1} / e)^{\mathbf{1 0}^{6}}$ randomly as an example.

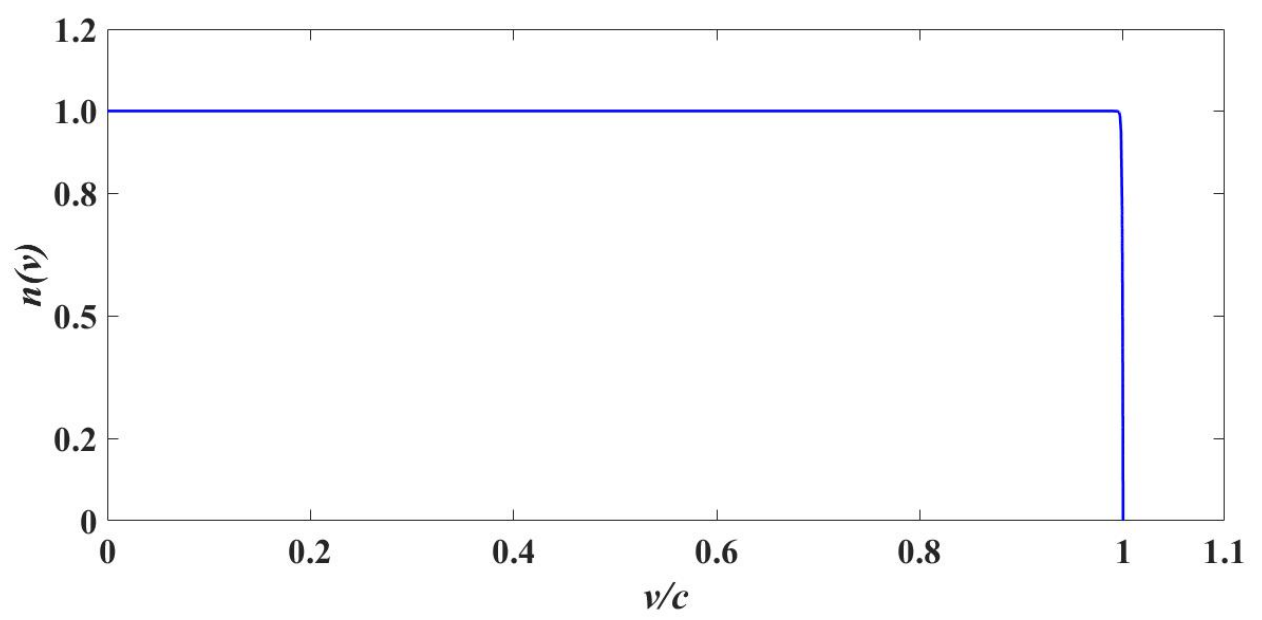

Fig. $1 n(v) \sim v$ curve when taking $Q=(1 / e)^{10^{6}}$

Thus, the time-space scaling factor limit and the particle's total energy limit are respectively

$$
\begin{aligned}
& \lim _{v \rightarrow c} \gamma=\lim _{v \rightarrow c} \frac{1}{\sqrt{1-v^{2} / k^{2}}}=\lim _{v \rightarrow c} \frac{1}{\sqrt{\left(\frac{1-\boldsymbol{v} / \boldsymbol{c}}{n}\right)\left(n+\frac{\boldsymbol{v}}{c}\right)}}=\sqrt{\frac{2 \ln Q}{Q-1}} \\
& \lim _{v \rightarrow c} E=\lim _{v \rightarrow c} \gamma m_{0} k^{2}=E_{Q G}=\frac{m_{0} c^{2}}{[1-0.5(Q-1) / \ln Q]} \sqrt{\frac{2 \ln Q}{Q-1}}
\end{aligned}
$$

\section{Modified arrival time lag effect}

It can be seen from Fig.1 that the modified particle's energy-momentum dispersion relation will return to the Lorentz case at low or medium energy. So next we will discuss the behavior of particles with ultrahigh energy.

When $\boldsymbol{v} \sim \boldsymbol{c}$ for an ultra-relativistic particle, it can be obtained from Eq. (17) that (set $c=1$ )

$$
n=\frac{1}{1-Q}\left(1-Q^{1-v^{2}}\right)=\frac{1}{1-Q}\left[1-Q^{(1+v)(1-v)}\right] \approx \frac{1}{1-Q}\left[1-Q^{2(1-v)}\right] \approx \frac{2 \ln Q}{Q-1}(1-v)
$$

Then 


$$
\begin{aligned}
\frac{E}{E_{Q G}} & =\frac{m_{0} k^{2}}{\sqrt{1-v^{2} / k^{2}}} /\left[\frac{m_{0} c^{2}}{[1-0.5(Q-1) / \ln Q]} \sqrt{\frac{2 \ln Q}{Q-1}}\right] \\
& =\frac{[1-0.5(Q-1) / \ln Q]}{\sqrt{2 \ln Q /(Q-1)}} \frac{n \boldsymbol{v} /(n-1+\boldsymbol{v})}{\sqrt{(1-\boldsymbol{v})(1+\boldsymbol{v} / n)}} \\
& \approx \frac{[1-0.5(Q-1) / \ln Q]}{\sqrt{2 \ln Q /(Q-1)}} \frac{2 \ln Q /(Q-1) v}{2 \ln Q /(Q-1)-1} \frac{1}{\sqrt{1-\boldsymbol{v}+\boldsymbol{v}(Q-1) /(2 \ln Q)}} \\
& \approx \frac{[1-0.5(Q-1) / \ln Q]}{\sqrt{2 \ln Q /(Q-1)}} \frac{2 \ln Q /(Q-1)}{2 \ln Q /(Q-1)-1} \frac{1}{\sqrt{(Q-1) /(2 \ln Q)+(1-\boldsymbol{v})}} \\
& \approx \frac{1}{\sqrt{2 \ln Q /(Q-1)}}\left[\sqrt{\frac{2 \ln Q}{Q-1}}-\frac{1}{2}\left(\frac{2 \ln Q}{Q-1}\right)^{3 / 2}(1-\boldsymbol{v})\right] \\
& =1-\frac{\ln Q}{Q-1}(1-\boldsymbol{v})
\end{aligned}
$$

Based on Eq. (20) we can obtain

$$
\frac{\boldsymbol{v}}{\boldsymbol{c}}=1-\frac{Q-1}{\ln Q}+\frac{Q-1}{\ln Q} \frac{E}{E_{Q G}}
$$

Eq. (21) shows that for an ultra-relativistic particle governed by Eqs. (14) and (17), its velocity is proportional to its energy.

Multiplying $m c^{2}$ on both sides of Eq. (21), we can obtain the equation for the photons

$$
\boldsymbol{p c}=E\left(1-\frac{Q-1}{\ln Q}+\frac{Q-1}{\ln Q} \frac{E}{E_{Q G}}\right)
$$

where $\boldsymbol{p}$ is the photon's momentum, and $E$ is the photon's energy.

If $E / E_{Q G} \sim 0$, then based on Eq. (22), we can obtain that

$$
\frac{\partial E}{\partial \boldsymbol{p}}=\frac{\boldsymbol{c}}{1-\frac{Q-1}{\ln Q}+\frac{Q-1}{\ln Q} \frac{E}{E_{Q G}}} \approx\left(1-\frac{Q-1}{\left.\ln Q \frac{E}{E_{Q G}}\right) \boldsymbol{c}}\right.
$$

Note that $Q \sim 0$ is used in Eq. (23). Now we apply Eq. (23) to study the photon's arrival time lag effect in astronomy. Based on Ref. [17], we can obtain the co-moving distance traversed by a photon, emitted at red-shift $z$ and traveling up to red shift 0

$$
x(z, E)=\frac{c}{H_{0}} \int_{0}^{z}\left[1-\frac{Q-1}{\ln Q}\left(\frac{E^{0}}{E_{Q G}}\right)\left(1+z^{\prime}\right)\right] \frac{d z^{\prime}}{\sqrt{\Omega_{m}\left(1+z^{\prime}\right)^{3}+\Omega_{\Lambda}}}
$$

where $E^{0}$ is the photon's energy measured at present, $\Omega_{\mathrm{m}}, \Omega_{\Lambda}$ and $H_{0}$ are the cosmological parameters evaluated today.

Then based on Eq. (24) we can obtain the photon's arrival time lag due to the modified energy-momentum dispersion relation in Eq. (14) as

$$
\Delta t=\frac{Q-1}{\ln Q} \frac{1}{H_{0}} \frac{E^{0}}{E_{Q G}} \int_{0}^{z} \frac{\left(1+z^{\prime}\right) d z^{\prime}}{\sqrt{\Omega_{m}\left(1+z^{\prime}\right)^{3}+\Omega_{\Lambda}}} \propto E^{0}
$$

In Ref. [16], the first-order approximation of Eq. (1) (that is, $\chi_{1}=1, \chi_{2}=0$ ) is taken 
to analyze the photon, and the corresponding arrival time lag was obtained as

$$
\Delta t=\frac{1}{H_{0}} \frac{E^{0}}{E_{L V}} \int_{0}^{z} \frac{\left(1+z^{\prime}\right) d z^{\prime}}{\sqrt{\Omega_{m}\left(1+z^{\prime}\right)^{3}+\Omega_{\Lambda}}} \propto E^{0}
$$

Comparing Eq. (25) with Eq. (26), we can obtain that

$$
E_{Q G}=\frac{Q-1}{\ln Q} E_{L V}
$$

Eq. (27) imply that the modified energy-momentum dispersion relation (i.e., Eq. (14)) is somewhat related to the rainbow model.

Importantly, it is well known that there are many experiments restricting the violation of Lorentz model, such as the experiments in Ref. [18-26], and these experiments' result imply the value of $Q$ is close to 0 (when $Q \equiv 0$ then $n \equiv 1$ ). In our previous work [27], we have restricted that $Q<(1 / e)^{10^{6}}$, and the corresponding time-space scaling factor $\gamma$ limit is bigger than 1414.2, while the above conclusion, i.e., for photons, it has $E_{\mathrm{QG}}=(Q-1) /(\ln Q) E_{\mathrm{LV}}$, indicates that the previous work on the restriction of $E_{\mathrm{LV}}$ can equally apply to constrain $E_{\mathrm{QG}}$ once we obtain the value of $Q$. But unfortunately, so far we still can't obtain the specific value of $Q$, for that the value of $Q$ can only be obtained in the experiment of particle with rest mass and ultrahigh energy. And we expect the specific value of $Q$ may be obtained in the future's neutrino experiment.

\section{Conclusions}

In this paper, we re-investigated the relationship between the speed of light and the symmetry of inertial systems based on the following three principles: (1)we can define the (proper) time in the whole space with a prescribed clock synchronization, (2)the time-space is uniform and the space is isotropic and (3)all the inertial systems are equivalent. We found that it was not necessary to restrict the speed of light to be a constant to meet the symmetry of inertial systems. And by imposing some special rules on the speed of light, we can construct a general coordinate transformation between inertial systems to meet the symmetry of inertial systems. Similar to the rainbow model, with a special assumption on the speed of light we also construct a non-Lorentz coordinate transformation between inertial systems to make the particle's energy have a limited value.

By analyzing the photon's arrival time lag effect in astronomy, we found that the particle's energy limit derived in our model is somewhat related to the particle's energy limit assumed in the rainbow model. That is, the first-order approximation of the rainbow model is somewhat related to the dispersion relations derived in our paper.

Compared with the rainbow model, Eq. (14) providing a new specific form for the generic modified dispersion relations (MDP) [28] has clear correction terms in physics, and the equation has just one undetermined parameter (the value of $Q$ ). Moreover, as Eq. (18) shown, the particle's total energy limit depends on the particle's rest mass, which is also different from the rainbow model or the usual theories of Quantum Gravity that the assumed or introduced "maximum energy or Planck energy" is independent of the particle's mass.

It should be noted that, although we construct a non-Lorentz transformation in above to make the particle's energy have a limited value, particles still cannot travel faster than the speed of light emitted by a stationary light source. And here we didn't 
claim certainly that $n$ deviates from 1 at the ultrahigh energy scale, but as the above shown, in theory and in no violation of the present experiments, it is possible that $n$ is not always equal to 1 . And similar to the rainbow model, if the value of $Q$ is not equal to 0 (if $Q \equiv 0$ then $n \equiv 1$ and Eq. (8) returns to the Lorentz case), then we will have to modify the current black hole model obtained from the general relativity theory, and therefore it may hopefully solve some problems faced by the current black hole model, which we will discuss in the next paper. 


\section{References}

[1]G A Camelia, J R Ellis, N E Mavromatos Internat. J. Modern Phys. A 12607 (1997).

[2]G. A Camelia Phys. Lett. B 510255 (2001).

[3]G P deBrito, P I C Caneda, Y M P Gomes, etc, arXiv:1610.01480 (2016).

[4]N Abbasvandi, M J Soleimani, S Radiman, W A T Abdullah Internat. J. Modern Phys. A 31 (23) 1650129 (2016).

[5]A Tawfik, H Magdy, A F Ali Phys. Part. Nucl. Lett. 1359 (2016) .

[6]S Doplicher, K Fredenhagen, J E Roberts Phys. Lett. B 33139 (1994).

[7]G A Camelia, J R Ellis, N E Mavromatos, etc Nature 393763 (1998).

[8]J Ellis, K Farakos, N E Mavromatos, etc Astrophys. J. 535 (1) 139 (2000) .

[9]C Liu, H Liu Astrophys. Space Sci. 357 (2) 114 (2015) .

[10]S Gangopadhyay, A Dutta Europhys. Lett. 115 (5) 50005 (2016) .

[11]Y Ling JCAP 070817 (2007) .

[12]A Awad, A F Ali, B Majumder J. Cosmol. Astropart. Phys. 1310052 (2013).

[13]J Bolmont, A Jacholkowska, J L Atteia, etc arXiv:astro-ph/0606725v6 (2007).

[14]N A Nilsson, M P Dabrowski Physics of the Dark Universe 18115 (2017) .

[15]H Xu, B Q Ma Astropart. Phys. 8272 (2016).

[16]H Xu B Q Ma Phys. Lett. B 760602 (2016) .

[17]U Jacob, T Piran J. Cosmol. Astropart. Phys. 0801031 (2008).

[18]M E Tobar, P Wolf, S Bize, etc Phys. Rev. D 81022003 (2010).

[19]M Nagel, S Parker, E Kovalchuk, etc Nat. Comm. 68174 (2015).

[20]H P Robertson Rev. Mod. Phys. 21378 (1949).

[21]R Mansouri and R U Sexl Gen. Rel. Grav. 8497 (1977).

[22]R Mansouri and R U Sexl Gen. Rel. Grav. 8515 (1977).

[23]R Mansouri and R U Sexl Gen. Rel. Grav. 8809 (1977).

[24]C Lämmerzahl, C Braxmaier, H Dittus, etc Int. Jour. Mod. Phys. D 111109 (2002).

[25]S Liberati Class. Quant. Grav. 3078 (2013) .

[26]F W Stecker, S T Scully, S Liberati, etc Phys. Rev. D 91 (4) 045009 (2015).

[27]J W Hu Physics Essays 303 (2017).

[28]G A Camelia, J R Ellis, N E Mavromatos, etc Nature 393763 (1998). 
Figures

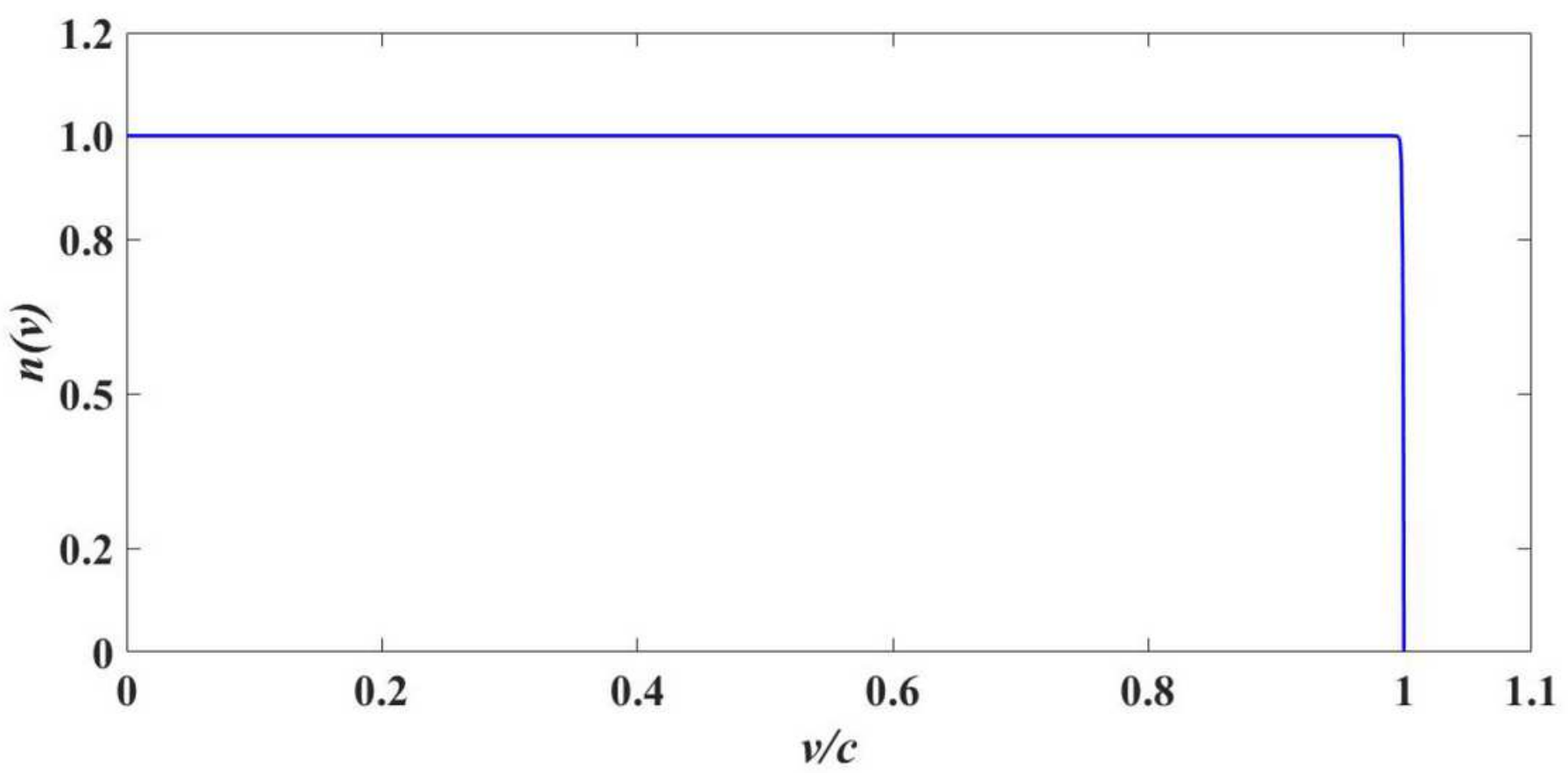

Figure 1

$\mathrm{n}(\mathrm{v}) \sim \mathrm{v}$ curve when taking $\mathrm{Q}=(1 / \mathrm{e}) 10^{\wedge} 6$ 